\title{
Reference Performance Test Methodology for Degradation Assessment of Lithium-Sulfur Batteries
}

\author{
V. Knap, (1) ${ }^{1, *, z}$ D-I. Stroe, ${ }^{1}{ }^{1}$ R. Purkayastha, ${ }^{2}$ S. Walus, ${ }^{2}$ D. J. Auger, ${ }^{3}$ A. Fotouhi, ${ }^{3}$ \\ and K. Propp ${ }^{3}$
}

\author{
${ }^{1}$ Department of Energy Technology, Aalborg University, Aalborg, 9000, Denmark \\ ${ }^{2}$ Oxis Energy Ltd, Culham Science Centre, Abingdon, Oxfordshire OX14 3DB, United Kingdom \\ ${ }^{3}$ Advanced Vehicle Engineering Centre, Cranfield University, Bedfordshire MK43 OAL, United Kingdom
}

\begin{abstract}
Lithium-Sulfur ( $\mathrm{Li}-\mathrm{S})$ is an emerging battery technology receiving a growing amount of attention due to its potentially high gravimetric energy density, safety, and low production cost. However, there are still some obstacles preventing its swift commercialization. Li-S batteries are driven by different electrochemical processes than commonly used Lithium-ion batteries, which often results in very different behavior. Therefore, the testing and modeling of these systems have to be adjusted to reflect their unique behavior and to prevent possible bias. A methodology for a Reference Performance Test (RPT) for the Li-S batteries is proposed in this study to point out Li-S battery features and provide guidance to users how to deal with them and possible results into standardization. The proposed test methodology is demonstrated for $3.4 \mathrm{Ah} \mathrm{Li-S} \mathrm{cells} \mathrm{aged} \mathrm{under} \mathrm{different} \mathrm{conditions.}$

(C) The Author(s) 2018. Published by ECS. This is an open access article distributed under the terms of the Creative Commons Attribution 4.0 License (CC BY, http://creativecommons.org/licenses/by/4.0/), which permits unrestricted reuse of the work in any medium, provided the original work is properly cited. [DOI: 10.1149/2.0121809jes]

(cc) BY
\end{abstract}

Manuscript submitted March 19, 2018; revised manuscript received May 8, 2018. Published May 26, 2018. This was Paper 538 presented at the New Orleans, Louisiana, Meeting of the Society, May 28-June 1, 2017.

Lithium-Sulfur (Li-S) is an emerging battery technology, which is gaining interest because of its high gravimetric energy density, increased safety, and expected low production cost. ${ }^{1-3}$ Because of these features, they might become an alternative to Lithium-ion (Li-ion) batteries and replace them in various areas, such as automotive, aerospace or personal equipment. However, the swift commercialization of $\mathrm{Li}-\mathrm{S}$ batteries is still hindered by their shortcomings of low coulombic efficiency, high self-discharge, and relatively rapid capacity fade. ${ }^{1,2}$ Nevertheless, Li-S batteries have already found areas of usefulness such as in high-altitude, long-endurance unmanned aerial vehicles. ${ }^{4}$

For product design, it is important to have a tool for comparison of performance and lifetime of various battery solutions. Moreover, it is required to have knowledge about the degradation of the battery in order to design safe and effective operational limits and control algorithms for the battery. Typically, standard battery accelerated degradation or lifetime tests are composed of an aging process (cycling or shelf idling) with periodical evaluation through a reference performance test (RPT). In the case of Li-ion batteries, there are several established test standards like ISO $12405-1 / 2,5,6$ IEC $62660-1 / 2,7,8$ which are summarized in the literature, ${ }^{9-11}$ advising how the $\mathrm{Li}$-ion batteries should be tested and evaluated. Similar guidelines are required for Li-S batteries. Unfortunately, Li-S chemistry with its specific mechanisms prevents the direct transfer of the methodologies from the Li-ion battery world. Not respecting these specific needs would lead to biased and incomplete results about the performance-degradation of the Li-S batteries.

The primary difference between Li-ion and the Li-S batteries are their charge and discharge mechanisms. Li-ion batteries undergo an intercalation process, wherein the $\mathrm{Li}$ ions travel from the cathode to the anode during charging and in the opposite direction during discharging. The charge and discharge processes are symmetrical and reversible, which gives them a consistent performance. ${ }^{12}$ Contrary to Li-ion batteries, Li-S batteries consist of a solution-based chemistry. When the Li-S battery is fully charged the sulfur at the cathode is in the dissolved form $\mathrm{S}_{8}{ }^{0}$ or in the solid $\mathrm{S}_{8}{ }^{0}$ and dissolved form $\mathrm{S}_{8}{ }^{2-} \cdot{ }^{13}$ During discharge, the reduction of $S_{8}$ undergoes a set of intermediate stages. At first long polysulfide chains of $\mathrm{Li}_{2} \mathrm{~S}_{8}$ and $\mathrm{Li}_{2} \mathrm{~S}_{6}$ are formed, and consequently, they are reduced into short polysulfide chains of $\mathrm{Li}_{2} \mathrm{~S}_{4}, \mathrm{Li}_{2} \mathrm{~S}_{2}$, and $\mathrm{Li}_{2} \mathrm{~S}$. During charge, the direction of the reactions is opposite: long chain polysulfides are formed from short chains. However, according to experimental observations,${ }^{14}$ the reaction pathways

*Electrochemical Society Student Member.

${ }^{\text {z} E-m a i l: v k n @ e t . a a u . d k ~}$ seems to be different for charge and discharge. Moreover, chemical precipitation takes place at the end of discharge for lithium sulfide $\left(\mathrm{Li}_{2} \mathrm{~S}\right)$ and at the end of charge for sulfur. Both lithium sulfide and sulfur are insulating and insoluble. Therefore, their precipitation causes both reversible and irreversible loss of the active material depending on the cycling. ${ }^{2}$ Another inherent mechanism of Li-S batteries is the polysulfide shuttle. Due to the high solubility of the long chain polysulfides, they diffuse toward the lithium anode, where they are reduced to short chain polysulfides. Then, the reverse flux is created by the high concentration of the reduced species at the anode and the reduced short chain polysulfides diffuse back to the cathode to be oxidized again. This shuttle parasitic reaction contributes to low Coloumbic efficiency, self-discharge and irreversible capacity loss. ${ }^{15}$

Based on proposed test methodologies for Li-ion batteries, degradation studies on Li-S batteries presented in literature, and especially our own experimental experience with Li-S batteries, an RPT dedicated to Li-S batteries is proposed in this work. Every step of the RPT is individually discussed and tailored to address Li-S specifics. An output of such an RPT is demonstrated by applying it to two cycle aging conditions for Li-S cells.

\section{Degradation Studies on Li-S Batteries}

Various types of studies on Li-S batteries can be found in literature, which includes some form of degradation tests and their evaluation. They can be sorted according to their objective into three main categories:

- cell development,

- mechanism investigations,

- modeling.

Cell development.- Studies focused on the cell development have usually limited scope about exploring the cells' degradation. They target mainly the comparison of the cycle life of a newly developed cell to the reference cell. Sometimes, the investigations go more in depth in order to explain the source of the prolonged life. The cells are usually cycled at only one, and rarely multiple, conditions. ${ }^{16-18}$

Mechanism investigations.-The goal of studies in this category is the investigation and understanding of the degradation mechanisms and influence of various factors and conditions. The literature provides multiple examples of different factors including but not limited to the effect of binders on battery performance and degradation investigated in Ref. 19. Brückner et al. studied the influence of C-rate, amount 


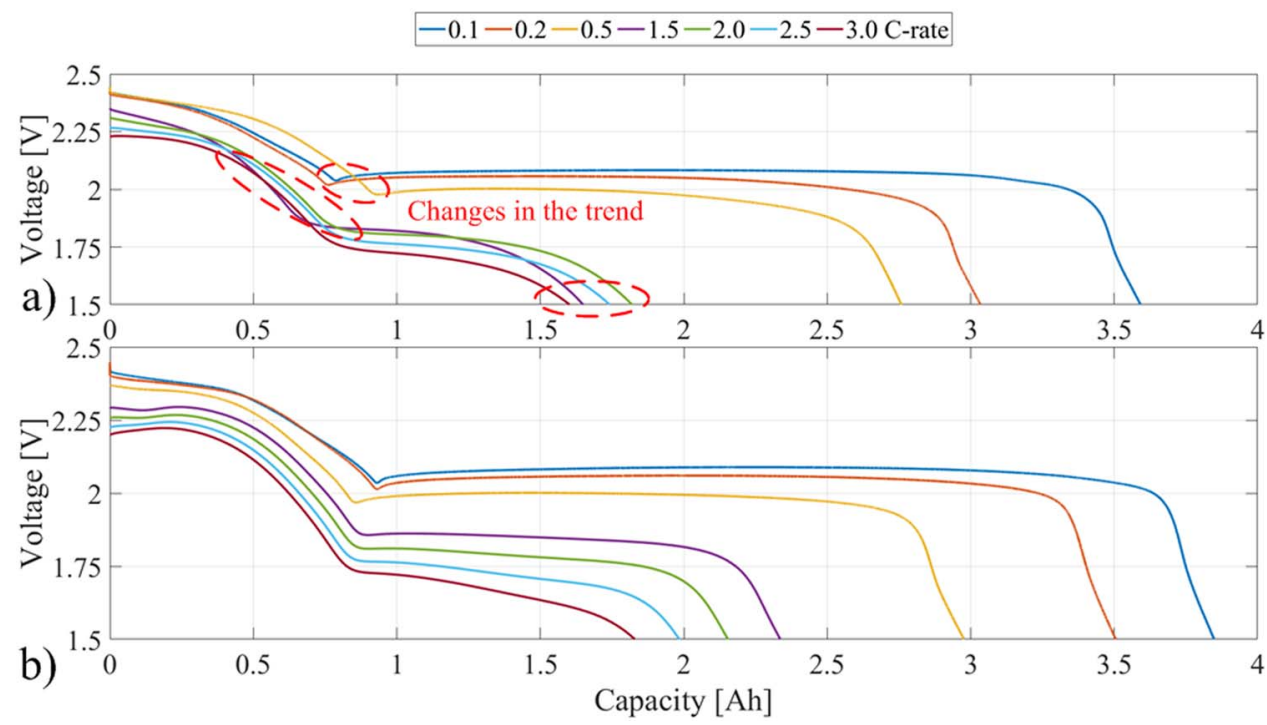

Figure 1. Voltage discharge curves for different C-rates: (a) without the pre-conditioning cycle, (b) with the pre-conditioning cycle before every charge (0.1 C-rate) and discharge (various C-rates).

of electrolyte and sulfur loading..$^{20}$ Moreover, the capacity fading mechanism of the cathode was analyzed in Ref. 21. The volumetric expansion during cycling was investigated in Ref. 22, where Lithium plating and the stripping process at the anode were identified as the main cause of Li-S cell swelling during aging.

Modeling.-The proposed models for the degradation of the Li-S batteries have typically one of the following roles: (i) a tool for investigation of the degradation mechanisms, ${ }^{23}$ (ii) being a part of a mechanistic model to reproduce the complex Li-S battery behavior ${ }^{24}$ or (iii) a separate component for prediction and simulation of the capacity fade. ${ }^{25}$

Analytical techniques.-Various analytical techniques have been applied to Li-S batteries which are summarized in Ref. 2, together with their benefits and limitations. However, the scope of battery degradation testing for the practical applications in this work is limited to applicable and measurable quantities of voltage, current and temperature, which can be obtained by the use of similar test equipment as needed for the degradation tests specified for Li-ion batteries in the literature. ${ }^{5-8,10,11}$

Galvanostatic techniques.-These are techniques where constant current cycling conditions are implemented i.e. full cell charge and discharge operations. These can be served as pre-conditioning cycles and can provide information about cell's charging and discharging energy, capacity, and efficiencies. Furthermore, the obtained voltage profiles can be analyzed for their change in the shape, or expressed as $\Delta \mathrm{Q} / \Delta \mathrm{V}$ vs $\mathrm{V}$ for an incremental capacity analysis ${ }^{26,27}$ or as $\Delta \mathrm{T} / \Delta \mathrm{V}$ vs $\mathrm{V}$ for thermal voltammetry analysis. ${ }^{28}$ Short current pulses applied to the battery are used to obtain the voltage response and subsequently determine the internal resistance of the battery. However, if the voltage limit is reached during the current pulse, the constant current (dis)charging mode has to switch to constant voltage mode. The user should always be careful when applying a constant charging voltage mode to the Li-S batteries due to the shuttle currents, which could result into an infinite charging of the cell and by that damaging it. The same applies to the constant current charging under specific conditions (low currents, high temperatures) where the charging time constraint should be included.

Potentiostatic techniques.-Cyclic voltammetry (CV) is a commonly used technique for the electrochemical characterization of the
Li-S cells. During CV, a constant ramp rate between two voltages is applied, which is then also reversed and the responding current is observed. Typically, a CV profile for Li-S batteries shows two pairs of redox peaks, which corresponds to voltage plateaus, obtained from the charging/discharging profiles. ${ }^{29}$ Another potentiostatic method is the direct shuttle current measurement, introduced in Ref. 15 and used for characterization and modelling in Ref. 30, in which the cell is kept at a constant voltage charging mode at the high voltage plateau until the current reaches the steady state and is matched by that the internal self-discharging shuttle current.

Electrochemical impedance spectroscopy (EIS).-For EIS measurements, the battery is excited by a sinusoidal current or voltage and its response to the other quantity is observed. The obtained impedance spectra are usually analyzed by fitting them to an electrical circuit model, in which particular elements are assigned to the specific electrochemical processes. However, for Li-S batteries, there is no consensus regarding the representation of the specific components. ${ }^{23}$

\section{Experimental}

Li-S long-life type cells, with a capacity of $3.4 \mathrm{Ah}$, provided by OXIS Energy ${ }^{31}$ were used for experiments. The measurements were performed using a Digatron BTS 600 battery test station. During the experiments, cells were kept in a temperature controlled environment. The temperature of $30^{\circ} \mathrm{C}$ is considered as the nominal value for comparison of the cells' performance. The nominal charging current was $0.34 \mathrm{~A}(=0.1 \mathrm{C}$-rate $)$ and the nominal discharging current was $0.68 \mathrm{~A}(=0.2 \mathrm{C}$-rate). The charging cut-off limits were $2.45 \mathrm{~V}$ or 11 hours. The discharging cutoff limit was $1.5 \mathrm{~V}$. A cycle, following these charging and discharging currents and limits is referred as nominal cycle.

Pre-conditioning cycles.-Due to the character of the Li-S chemistry, the actual performance of the cell is highly dependent on its previous history, ${ }^{13,25}$ which is the so-called 'cumulative history' effect. This can be illustrated by the discharge capacity test for different C-rates shown in Fig. 1. For the first cell, the discharge procedure was as follows: (i) charge to $2.45 \mathrm{~V} / 11$ hours by 0.1 C-rate, (ii) discharge to $1.5 \mathrm{~V}$ by a specific C-rate, (iii) relaxation 15 minutes and (iv) discharge to $1.5 \mathrm{~V}$ by $0.2 \mathrm{C}$-rate. This procedure was repeated for various C-rates from $0.1 \mathrm{C}$ to $3 \mathrm{C}$. As it is visible in Fig. 1a, the discharge curves do not have a homogenous trend between 


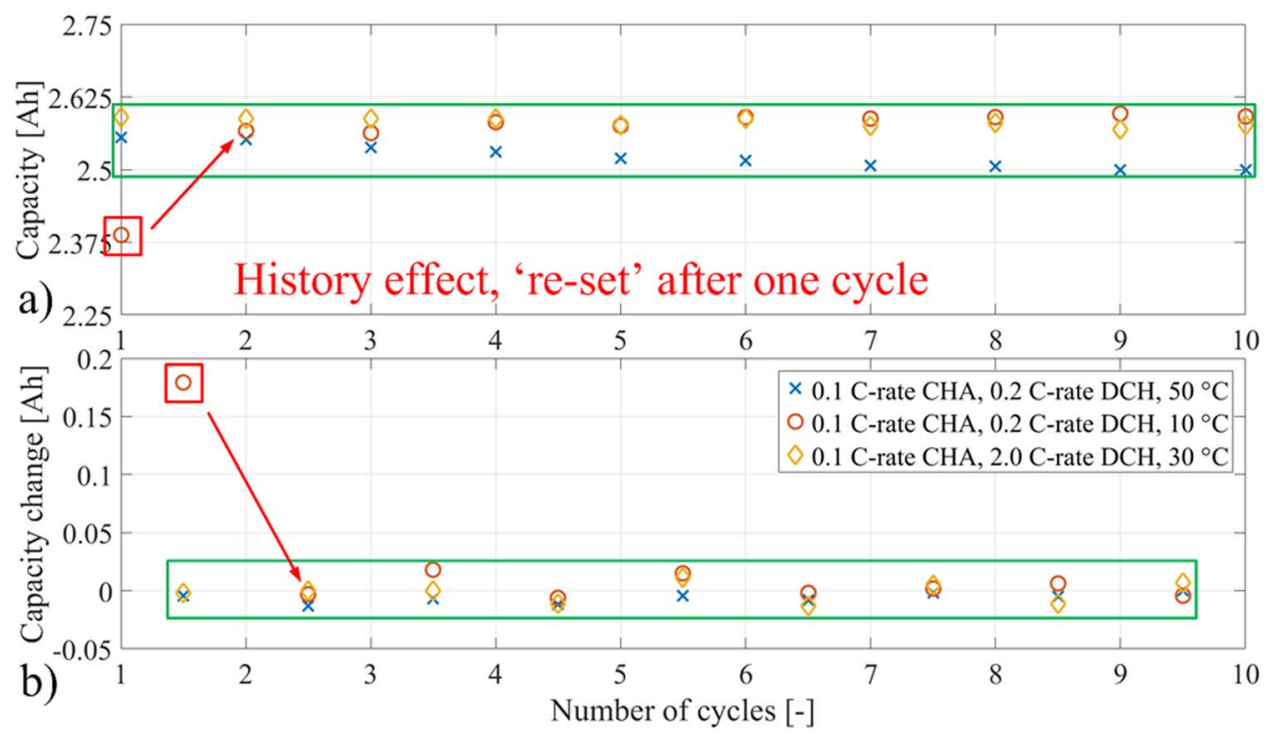

Figure 2. Evaluation of the nominal cycles at $30^{\circ} \mathrm{C}$, after cycling at different conditions; a) capacity obtained from each cycle, b) change in the capacity between the cycles.

each other. The discharged capacity is not always in the order of the applied current, as the cell discharged by $1.5 \mathrm{C}$ has lower capacity than cells discharged by 2 or $2.5 \mathrm{C}$. The procedure for the second cell was modified by inserting one nominal cycle $(0.1 \mathrm{C}$ charging, $0.2 \mathrm{C}$ discharging) before every charging step of the discharge capacity test procedure. The resulting discharge curves for the second cell are presented in Fig. 1b show a relatively homogenous trend for the different discharging C-rates. Therefore, a pre-conditioning cycle is required in order to obtain repeatable results at common reference state of the cells. The reason for this behavior is believed to be the precipitation of lithium sulfide. Lithium sulfide can precipitate at different rates and the precipitates can be of different morphology when different discharge rates are applied. ${ }^{32}$ More importantly, all the lithium sulfide may not re-dissolve back on charge, leading to a temporary 'loss' of capacity. By adding an additional nominal cycle, we allow complete redissolution to occur, and essentially the cell 'resets' correctly to allow for accurate measurements.

The required number of pre-conditioning cycles might vary with the specific cell composition, its size and the conditions at which the cell is exposed to, both environmental and operational. In order to determine this number of cycles, the considered $3.4 \mathrm{Ah}$ cell was exposed to 10 cycles at different specific conditions (various current and temperature), followed by 4 hours of temperature stabilization at $30^{\circ} \mathrm{C}$ and subsequent 10 nominal cycles. The specific cycling conditions were selected to match the limiting conditions of the future considered degradation tests. In our case it was chosen: nominal currents at $50^{\circ} \mathrm{C}$; nominal currents at $10^{\circ} \mathrm{C}$; and $0.1 \mathrm{C}$ charging, $2.0 \mathrm{C}$ discharging currents at $30^{\circ} \mathrm{C}$. The obtained capacities from the nominal cycles at $30^{\circ} \mathrm{C}$ are shown in Fig. 2, together with the capacity change between two consecutive cycles. The capacity can be significantly different at the first cycle, but since the second cycle, the changes in the capacity between the cycles are only minor. Therefore, it is suggested that only one pre-conditioning cycle is needed and the second cycle can be already used for the capacity evaluation.

Capacity measurement.-Capacity measurement of the cell is done by using specific currents to obtain the cell capacity, energy, and efficiency at the specific C-rates. For our procedure, we considered only nominal currents due to time constraints. The advantage of this consideration is that the capacity measurement and the preconditioning cycle are done in the same cycle. Therefore, the next step of the RPT can follow directly. The capacity obtained during the discharge is used further on for computing the SOC of the cell. The capacity measurements can be expanded by using additional C-rates; however, then adding pre-conditioning cycles before or after (due to following measurements) should be considered, together with the total time required for the RPT and also additional degradation of the cell during the RPT. For example, if the discharge capacity test of $1 \mathrm{C}$-rate is added, it will demand $10+1=11$ hours for only the additional discharge test and also $10+5=15$ hours for another pre-conditioning cycle, which will prolong the RPT by 26 hours.

Power and resistance measurement.-The resistance, together with the pulse power capability, is recommended to be measured through either the hybrid pulse power characterization (HPPC) test $t^{5,6,10}$ or through the pulse train. ${ }^{7,9,11}$ The HPPC test was designed for the automotive industry to evaluate the battery dynamic power capability during high pulse discharge ( 10 seconds, maximum discharge current), followed by a short relaxation ( 40 seconds) and the regenerative charge pulse (10 seconds, 0.75 of the maximum discharge current). ${ }^{5}$ The pulse train consists of a set of charging and discharging current pulses following each other from the smallest or largest current values. The pulse is followed by another pulse with the opposite polarity in order to maintain the SOC constant. The advantage of the pulse train is that it retrieves information including the current dependence of the internal resistance, which is especially useful when a parameter identification procedure is applied to the pulses in order to obtain values for the parameters of a battery electrical circuit model.

Three different values of current for charging and discharging were considered to be sufficient in order to obtain the current dependence of the battery parameters. The Li-S battery is more of a high energy than a high power cell due to its relatively high resistance i.e. the utilization of cell under investigation was significantly reduced at high C-Rates. Thus, even though the cell under investigation was capable of $3 \mathrm{C}$ continuous discharge, the total obtained capacity is significantly reduced (Fig. 1b) at this C-rate. Then, the discharging mode would very often be limited by voltage rather than current. Moreover, it is not a current level expected to be experienced by a single cell at the considered battery application of electric vehicles. Therefore, the current of $1 \mathrm{C}$ was selected as a compromise to be closer to the realistic operation scenarios. As mentioned before, the charging process of the $\mathrm{Li}-\mathrm{S}$ battery is not symmetric to the discharging process, the charging pulse currents were selected to be smaller (half in our case) according to the charging capability of the cell. Finally, the applied currents were $0.1,0.2$ and $0.5 \mathrm{C}$-rate for charge pulses and $0.2,0.5$ and $1 \mathrm{C}$-rate for discharge pulses. 
Table I. Settling time after pulses at various SOC levels.

\begin{tabular}{ccccccccrrr} 
SOC [\%] & 90 & 80 & 70 & 60 & 50 & 40 & 30 & 20 & 10 \\
\hline Pulse current & & & \multicolumn{7}{c}{ Settling time [seconds] } \\
\hline 1 C-rate DCH & 108 & 480 & 586 & 562 & 569 & 500 & 452 & 311 & 297 \\
0.5 C-rate CHA & 622 & 430 & 89 & 200 & 353 & 179 & 316 & 244 & 173
\end{tabular}

The relaxation period between the pulses for the $\mathrm{Li}$-ion batteries is recommended to be 10 minutes, ${ }^{7}$ unless the cell temperature is still higher than $2^{\circ} \mathrm{C}$ of target test temperature, then the cell can be cooled down or the relaxation period can be prolonged. For the Li-S battery, we have first extended the relaxation period to 15 minutes and performed the preliminary pulse train test from $90 \%$ SOC to $10 \%$ SOC at $25^{\circ} \mathrm{C}$. For obtaining the necessary relaxation time between the pulses, the following assumption was made in order to compute the settling time: the system is sufficiently relaxed when the voltage reaches $95 \%$ of a quasi-steady state voltage value (at 15 minutes of the relaxation after the pulse) from the initial voltage drop value. Only the worst case of the current was considered, i.e. $0.5 \mathrm{C}$ for charging and $1.0 \mathrm{C}$ for discharging. The obtained settling time values are summarized in Table I. At very high SOC, the polysulfide shuttle changes the character of the recovery voltage and therefore, the settling time at $90 \%$ of SOC varies significantly from the other SOC levels. The average values for the interval from 80 to $10 \%$ SOC were computed to be 470 seconds $=7.83$ minutes for $1.0 \mathrm{C}$ discharge pulses and 248 seconds $=$ 4.13 minutes for $0.5 \mathrm{C}$ charge pulses. Rounding the numbers up to 10 and 5 minutes for discharging and charging consequently provides a margin to ensure that the cell should be sufficiently relaxed and the values should be valid also for the SOC levels at the neighboring temperature levels (such as 20 or $30^{\circ} \mathrm{C}$ ) with a lower rate of the polysulfide shuttle.

When 10 and 5 minutes relaxation periods between pulses were applied, there has been observed a steep voltage drop at $100 \%$ and $90 \%$ SOC due to the prevalence of polysulfide shuttle. Moreover, the 'equilibrium point' ( $=$ the peak point between voltage recovery after discharge and voltage decay due to the self-discharge) was present relatively early. Therefore, the relaxation periods can be much shorter, which is also preferable due to the smaller shift of the SOC caused by the self-discharge. It has been assumed that only half of the relaxation periods used for other SOC levels ( 5 minutes for discharging and 2.5 minutes for charging pulses) is sufficient for $90 \%$ SOC and quarter of it (2.5 minutes for discharging and 1.25 minutes for charging pulses) is enough for $100 \%$ SOC.
After the discharging steps between different SOC levels and before the first pulse, there is a requirement for an additional relaxation time to allow the cell to reach an equilibrium state. However, for some SOC levels that would mean a relaxation in range of hours, which would considerably prolong the overall test procedure. ${ }^{33}$ Therefore, a 30 minutes long relaxation period is considered sufficient to reach a quasi-equilibrium state for the cell before the pulse train procedure. The applied pulse train for 0 to $80 \%$ SOC is illustrated in Fig. 3. Again, due to the self-discharge at higher SOC levels, the relaxation is shortened to 15 minutes at $90 \%$ SOC and it is only 1.5 minutes at $100 \%$ SOC.

The last step is to correctly determine the discharging step between the SOC levels. Due to inequality between the charging and discharging pulses, the SOC shifts down by $0.75 \%$ per pulse train, except at $100 \%$ SOC, where the $0.5 \mathrm{C}$ charging pulse is omitted, as the limiting voltage is already reached by $0.2 \mathrm{C}$ charging pulse; and therefore the SOC shifts down by $1.2 \%$. The discharging steps should be adjusted to account for this SOC shift. Moreover, the approximate self-discharge can be estimated by the Li-S self-discharge model ${ }^{30}$ with a consideration of a fresh cell with 3.4 Ah capacity. The pulse train procedure at $100 \%$ lasts 14 minutes $(1.5$ minutes relaxation period before the first pulse, $2^{*} 0.5$ minutes charging pulses, $3 * 0.5$ minutes discharging pulses, $2 * 1.25$ minutes relaxation after charging pulses and $3 * 2.5$ minutes relaxation after discharging pulses), which results in a loss of $0.78 \%$ SOC. Therefore, immediately after the pulse train at $100 \%$ SOC level, the actual SOC would be rather $98 \%$. So the discharge to $90 \%$ SOC level can be reduced down to step of $7.5 \%$ SOC, to account for the previously described occurrences and for the self-discharge during this discharging step. A similar procedure can be applied to compensate for the self-discharge during the pulse train at $90 \%$ SOC; however, its effect is under $1 \%$ SOC and it is considered insignificant to be dealt with. The last effect, which can be considered for adjustment of the discharging steps between SOC levels, is the charge recovery effect. ${ }^{34}$ As the cell is relaxed between the discharging steps, its effective capacity is higher than during the continuous discharge. Therefore, the discharging step between $10 \%$ and $0 \%$ SOC should

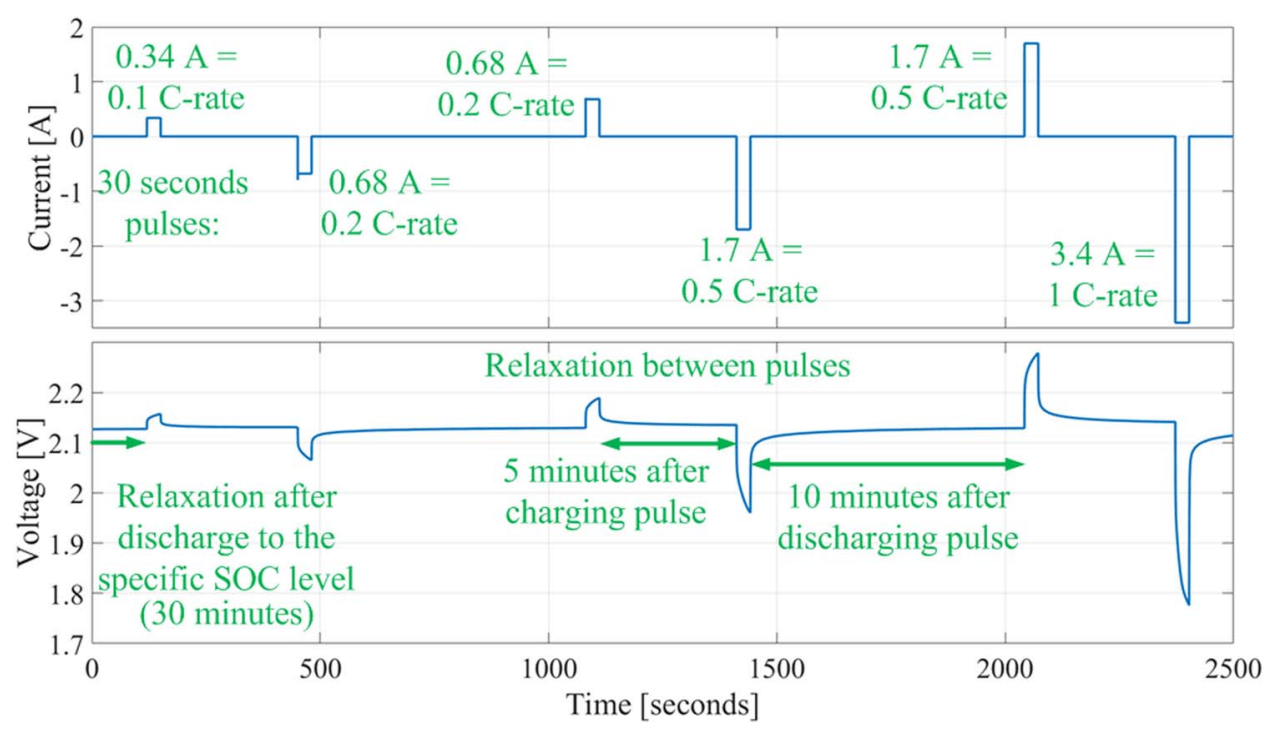

Figure 3. Illustration of the applied pulse train procedure for SOC levels between 0 and $80 \%$ SOC. 
Table II. Specifications for pulse train procedure at various SOC levels.

SOC level [\%]

Discharging step to the SOC level

Relaxation before the pulse train [min]

Relaxation after charging pulse [min]

Relaxation after discharging pulse [min]
100

1.5

1.25

2.5
90

$7.5 \%$

15

2.5

5
$80-10$

$9.25 \%$

30

5

10
0

to $1.5 \mathrm{~V}$

30

5

10 be controlled rather by discharging to cutoff voltage limit of $1.5 \mathrm{~V}$ than controlled by the amount of discharged SOC, in order to bring the cell to the state when is actually no charge available. The summary of different settings according to SOC levels are presented in Table II.

Shuttle current measurement.-The polysulfide shuttle is a unique mechanism, which has no equivalent within classical Li-ion batteries; nevertheless, for Li-S batteries, it is very important, as it is related to the self-discharge, degradation, columbic efficiency and possibly also to the safety. Moy et al. ${ }^{15}$ introduced the methodology for the measurement of the polysulfide shuttle current, which is based on constant voltage charging until the external current reaches a steady-state and which indicates that it has equalized with the internal shuttle current.

The procedure follows the downward SOC direction, so at first, the cell has to be charged. Then the cell is discharged to the target SOC level and it is relaxed until the voltage equilibrium is reached. The voltage equilibrium, illustrated in Fig. 4, is understood to be the peak voltage value, which occurs between increasing voltage in the recovery period immediately after the interruption of discharging current; and decreasing voltage due to self-discharge in pro-longed relaxation. In practice, the voltage equilibrium can be detected by the voltage falling under the threshold from the maximum voltage value, where the threshold is set with respect to the measurement accuracy and noise. When the threshold is crossed, this voltage value is used as the limit for constant voltage charging, which lasts until the current reaches steady-state value, typically limited by time. Afterward, it is followed by the discharging to the next investigated SOC level.

The procedure and results for the shuttle current measurement on 3.4 Ah Li-S cells have been already presented in Ref. 30. From that work, it is known a period of two hours of constant voltage charging is enough for these cells to reach steady-state. Moreover, the voltage threshold applied in a Digatron BTS 600 battery test station is $0.6 \mathrm{mV}$. It is considered that three different SOC levels for the shuttle current measurement should be enough, together with the fourth level with known zero current, to use the results for fitting and deriving a relation of the shuttle current against SOC or open circuit voltage (OCV). The SOC levels, $98 \%, 94 \%$, and $88 \%$ were considered in order to get the shuttle current distribution over a wide SOC/OCV region, but not to pro-long the test unnecessarily. The shuttle current measurement procedure is illustrated in Fig. 5.

Summary of the proposed RPT.-The proposed RPT methodology for the Li-S batteries is composed of the previously introduced steps: pre-conditioning cycles, capacity measurement, power and resistance measurement and shuttle current measurement. The specific steps were adjusted according to the requirements of the specific cell type, in our case 3.4 Ah Li-S pouch cell from OXIS Energy. It has been found that 4 hours temperature stabilization and one pre-conditioning cycle ( $0.1 \mathrm{C}$ charging, $0.2 \mathrm{C}$ discharging) are sufficient to 'reset' the history of the cell and obtain comparable results from the RPT procedure after exposing the cell to cycling at three extreme conditions. The capacity measurement is performed only for the nominal currents of $0.1 \mathrm{C}$ for charging and $0.2 \mathrm{C}$ for discharging, which allows moving to the next step of power and resistance measurement without an additional pre-conditioning cycle in between. The power and resistance measurement are performed by the pulse train starting from $100 \%$ SOC and continue down to $0 \%$ by the steps of $10 \%$. The pulses are asymmetric for charging and discharging. An additional step of the RPT for the Li-S batteries is represented by the shuttle current measurement, which allows quantifying the shuttle in a straightforward way and by that provide information about the self-discharge and the cell degradation rate. The Li-S battery cell voltage profile during the proposed RPT procedure is shown in Fig. 6. The specific parameters of the applied RPT are presented in Table S.I in the Supporting Information.

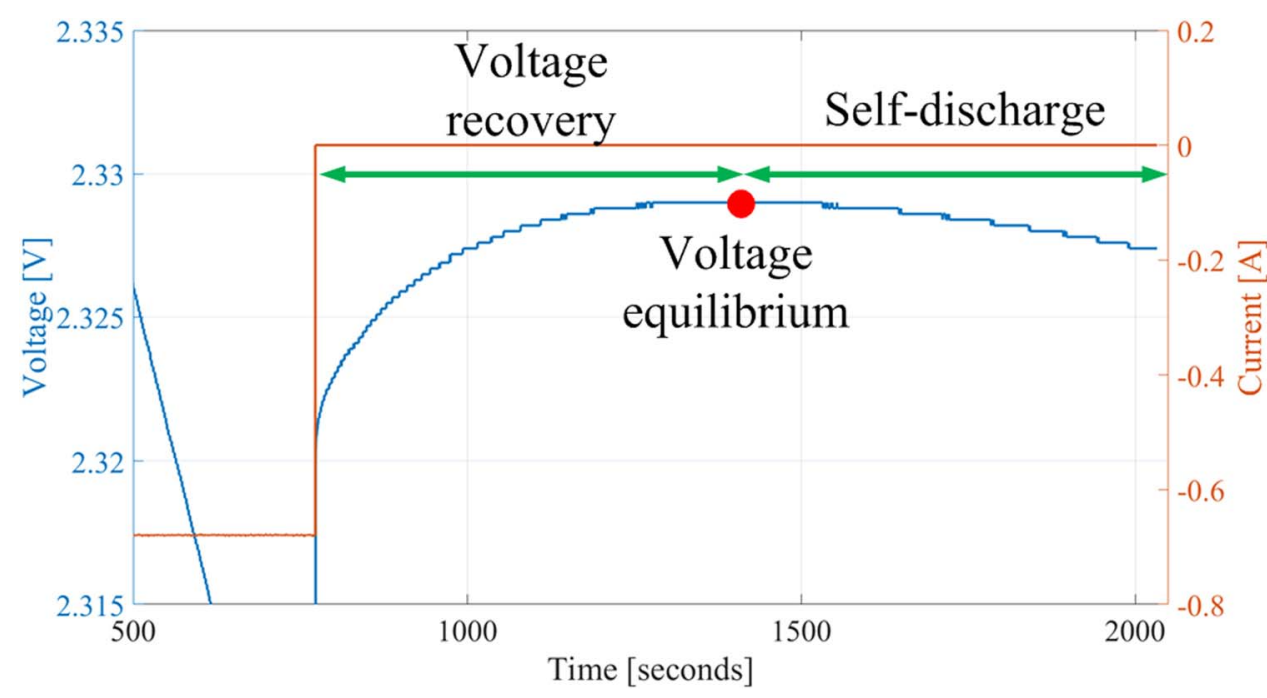

Figure 4. Illustration of the voltage equilibrium point during a relaxation period after a discharge at the high voltage plateau of Li-S batteries. When the discharging current is interrupted, at first the voltage rises during "voltage recovery period," then the voltage peak is reached when the influence of recovery and self-discharge on voltage is equalized. Afterward, the voltage starts to decay due to the prevailing effect of the self-discharge on the voltage. 

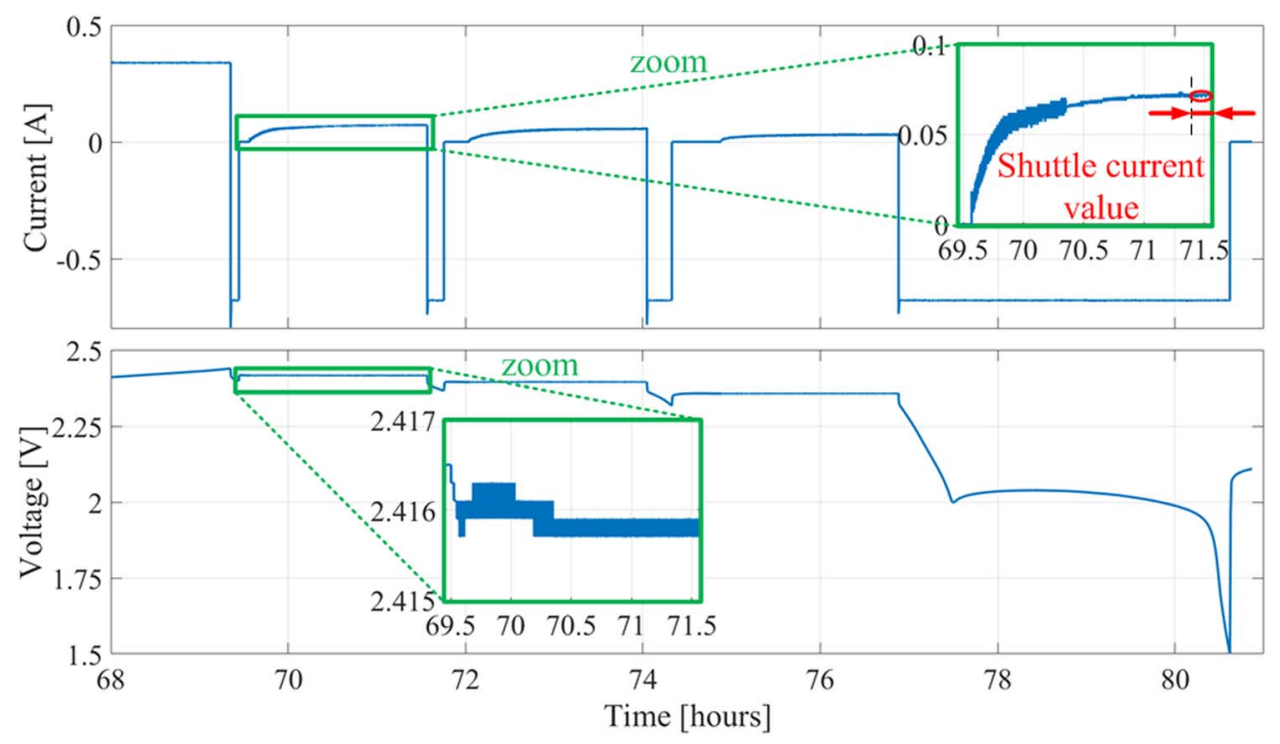

Figure 5. Illustration of the shuttle current measurement procedure.

The time requirement was a criterion used in designing the RPT methodology. The use of low currents results in a relatively long time required for charging and discharging. Specifically, in this case, where nominal currents are $0.1 \mathrm{C}$ for charging and $0.2 \mathrm{C}$ for discharging, the nominal cycle lasts 15 hours. Throughout the proposed procedure, there are four full cycles, which then take 60 hours. The relaxation periods during the resistance measurement, summarized from Table II, last for approximately 12 hours. The constant voltage charging during shuttle current procedure takes six hours (three SOC levels, each with two hours) and the relaxation there is estimated to be around three hours. Consequently, the RPT procedure is expected to last around 81 hours. This is the resulting time when the capacity is measured for only one C-rate and only one pre-conditioning cycle is needed. Thus, the limited power capability in terms of only low applicable currents indicates another limitation for Li-S cells, which is a high cost of testing due to its longtime requirement.

If needed, the RPT can be expanded by adding, for example, EIS or $\mathrm{CV}$ as extra steps, which would result in extra cycles and time demand. Alternatively, to avoid this, the shuttle current measurement procedure can be periodically (e.g. every second RPT) replaced by either EIS or CV. Another beneficial measurement to add would be thickness measurement of the cell as described in Ref. 22. A laser thickness gauge can be used for the measurement during cycling. However, for a periodical RPT procedure, the use of a regular micrometer would be sufficient.

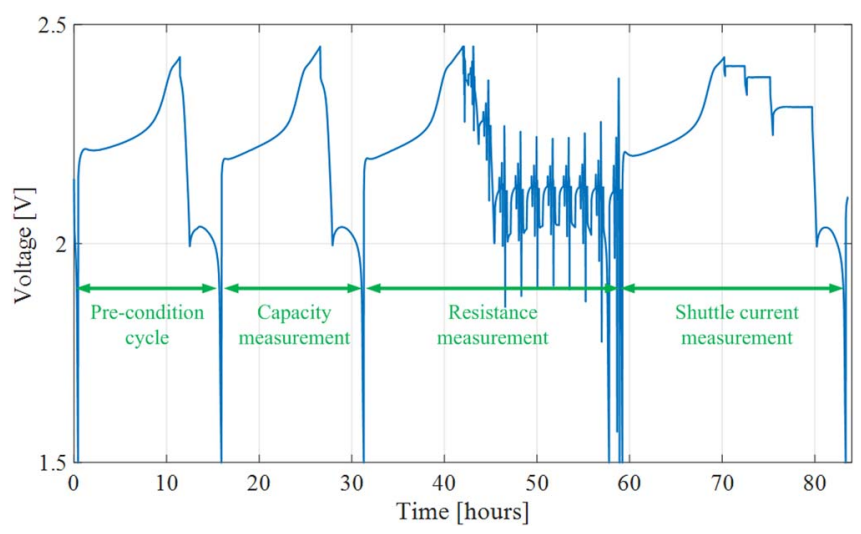

Figure 6. Illustration of the complete RPT procedure for the Li-S batteries.
RPT applied to the Li-S cells.-In order to illustrate the use and the outcome of the proposed RPT, the Li-S cells were subjected to cycle aging and every 20 cycles the proposed RPT procedure was performed. The considered cycling conditions for each test case (TCs) were:

- TC1: $0.1 \mathrm{C}$ charging to $2.45 \mathrm{~V}$ or 11 hours, $0.2 \mathrm{C}$ discharging to $1.5 \mathrm{~V}$ at $30^{\circ} \mathrm{C}$

- TC2: $0.1 \mathrm{C}$ charging to $2.45 \mathrm{~V}$ or 11 hours, $0.5 \mathrm{C}$ discharging to $1.5 \mathrm{~V}$ at $30^{\circ} \mathrm{C}$

The absolute discharge capacities obtained during cycling and during the RPT are presented in Fig. 7. As expected, the capacities from cycling and from the RPT for TC1 are matching, because the capacity test is performed at the same conditions as cycling (0.1 C-rate charging, $0.2 \mathrm{C}$-rate discharging, $\left.30^{\circ} \mathrm{C}\right)$. However, at TC2 there is an apparent difference between cycling and RPT capacities, which demonstrates the need of the RPT at common conditions. The cell tested under TC2 conditions is cycled with a higher current of $0.5 \mathrm{C}$-rate in comparison to TC1. Therefore, the cycling discharge capacity of TC2 is limited mainly due to the limited mass transfer. ${ }^{35}$ This limitation is believed to be caused by an increased amount of anion species such as $\mathrm{S}_{4}{ }^{2-}$ and $\mathrm{S}_{2}{ }^{2-}$ being trapped in the separator, based on a larger concentration gradient caused by a higher discharge current. $^{36}$

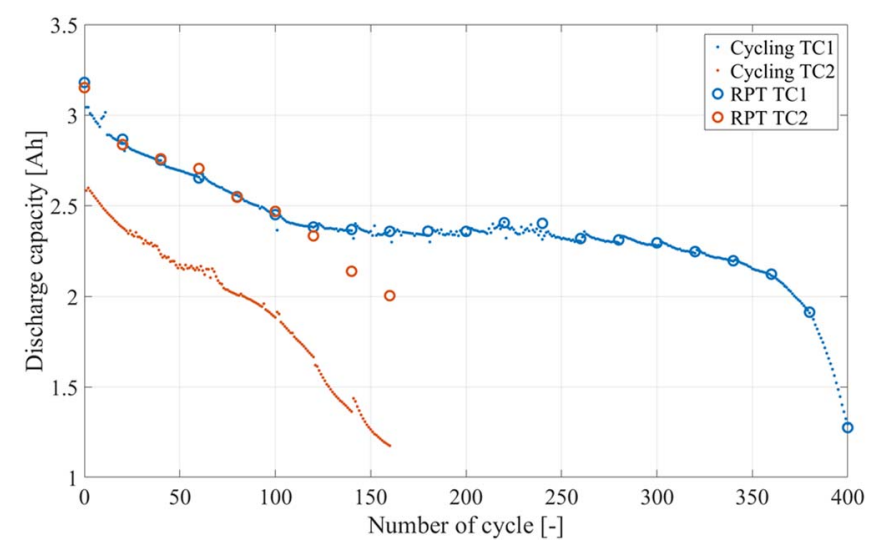

Figure 7. The discharge capacity of the Li-S cells retrieved from the cycling and from the RPTs. 


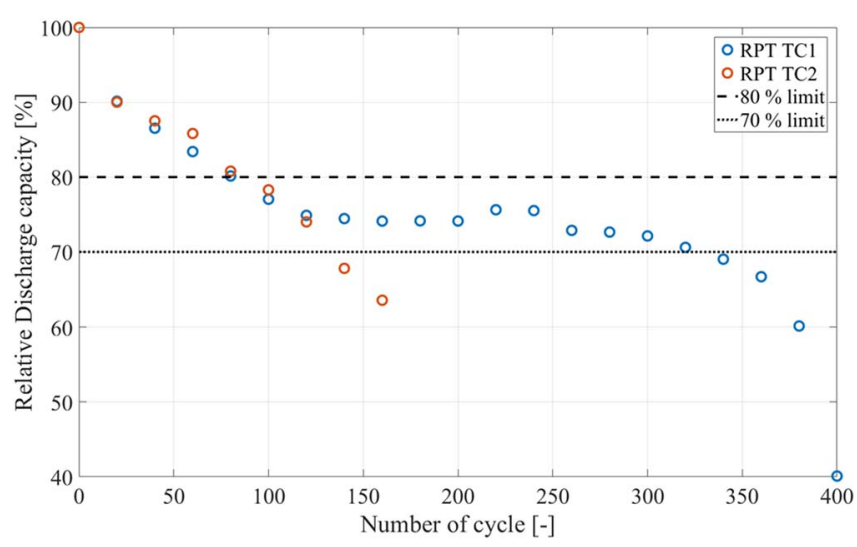

Figure 8. The relative discharge capacity of the Li-S cells retrieved from the RPTs with illustrated end-of-life limits.

Relating the cycling discharge capacities between different TCs might be very difficult, especially without any reference point. It is possible to relate the discharge cell capacities at a different temperature or discharge rate by a coefficient, but as it is shown in Fig. 7, this relation changes during the aging. Thus, RPT capacities are used to obtain a fair comparison of capacity decay during aging of cells under various conditions. One can observe from Fig. 7 and Fig. 8 that the RPT capacity had a similar trend for both TCs for the first 120 cycles. After 140 cycles, the capacity reduction is more significant for the cell tested under TC 2 conditions. The end-of-life criterion is often considered to be $80 \%$ of the initial cell's capacity for Li-ion batteries. By considering the same limit for the Li-S batteries, the useable cycle life of the tested cells is 80 cycles. However, the Li-S batteries have a higher gravimetric capacity than Li-ion batteries, ${ }^{37}$ so they are still able to deliver the similar capacity as Li-ion batteries while experiencing significant capacity fade. Therefore, the end-of-life criterion for the Li-S batteries in terms of capacity can be lower, e.g. $70 \%$ of the initial capacity, which results in extending the useful cycle life to approximately 130 cycles for TC2 and 330 cycles for TC1.

The RPT results for TC1 and TC2 are presented in Fig. 9 and Fig. 11, respectively. Due to a relatively large amount of available data, it was selected to show the capacity fade for every 20 cycles, while the shuttle current, the resistance, and power evolution are shown for every 40 cycles; the resistance was determined for a one second 0.2 C-rate discharging current pulse, while the power was computed for a 30 seconds $1 \mathrm{C}$-rate discharging current pulse. The capacity fade $\left(\mathrm{Cap}_{\text {fade }}\right)$ was computed as follows:

$$
\mathrm{Cap}_{\text {fade }}=\left(\mathrm{Cap}_{\mathrm{BOL}}-\mathrm{Cap}(\mathrm{n})\right) / \mathrm{Cap}_{\mathrm{BOL}}{ }^{*} 100
$$

Where Cap $_{B O L}$ is the cell's capacity at the beginning of life (from the RPT performed before cycling) and $\operatorname{Cap}(n)$ is the capacity obtained from the RPT after $n$ cycles.

In a Li-S cell a multiple aging mechanisms occur at the same time, such as change of the cathode structure during cycling, irreversible deposition of solid $\mathrm{Li}_{2} \mathrm{~S}$ species on the cathode surface causing its clogging, formation of mossy Lithium and anode degradation, electrolyte depletion and many more, which degrade the cell performances with the time. Decoupling of these effect is still challenging, nevertheless, the literature present many examples with studies of Li-S cells aging. ${ }^{20,38,39}$ In this work we can clearly observe capacity fade with cycling, which especially enhanced at higher discharge current. It is difficult though to understand what is exact cause of that fade. The purpose of this study was rather using the RPT protocol for characterization, rather than fundamental investigation of the cause of the capacity fade.

Aging at the TC1 conditions resulted into a longer cycle life. The shape of the capacity fading curve, presented in Fig. 9a), indicates an influence of the fast (in the beginning) and slow (in the middle) capacity fading processes, which were described in Ref. 40. Moreover, an additional rapid capacity fading in interval of 360 to 400 cycles is captured. The measured values of the shuttle current are shown
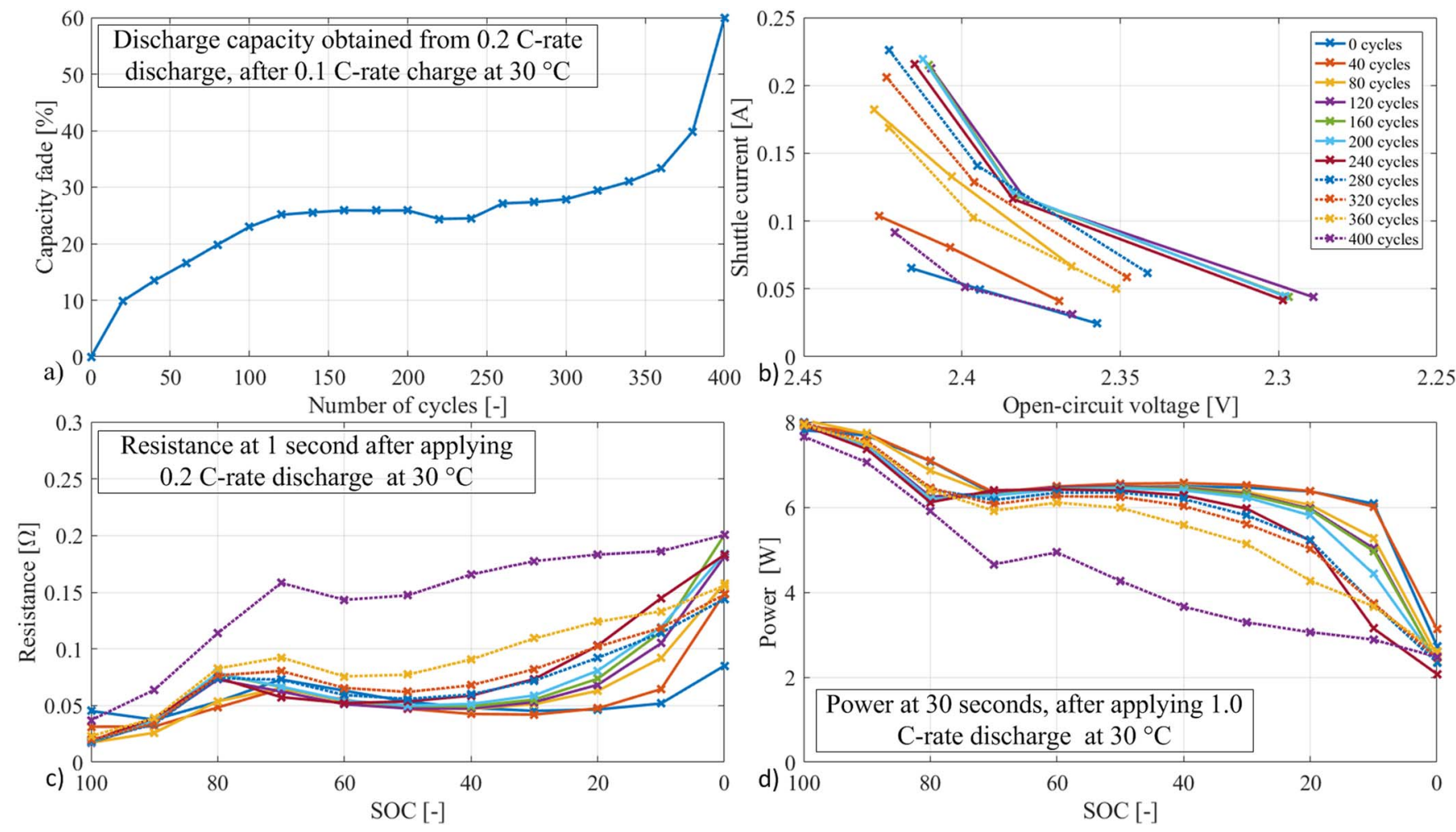

Figure 9. Evolution of the performance parameters obtained during TC1 aging ( $0.1 \mathrm{C}$-rate charging to $2.45 \mathrm{~V}$ or 11 hours, $0.2 \mathrm{C}$-rate discharging to $1.5 \mathrm{~V}$ at $\left.30^{\circ} \mathrm{C}\right)$. 


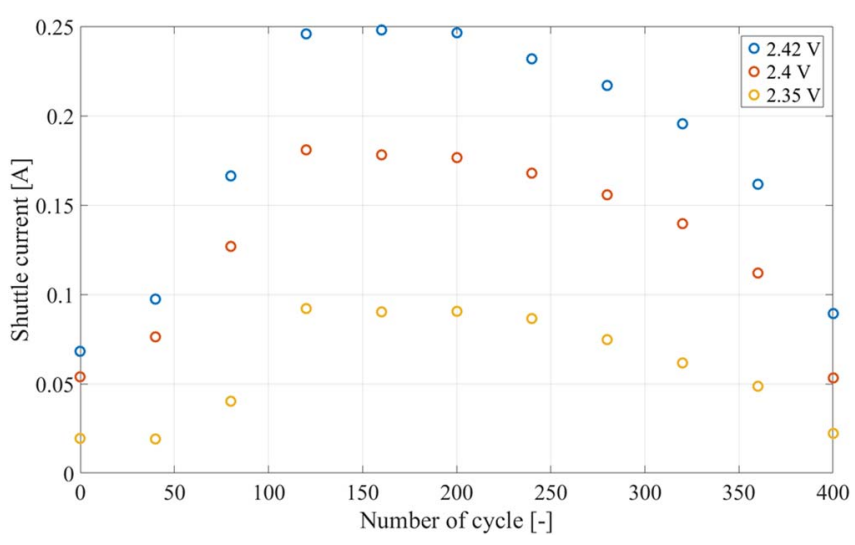

Figure 10. Interpolation of the measured shuttle current for open-circuit voltage values of 2.42, 2.4 and $2.35 \mathrm{~V}$ for TC1 and their evolution during cycling.

in Fig. 9b). One can see that the shuttle current is at first increasing during aging until 120 cycles and then from 200 cycles it is again decreasing. For a more intuitive comparison, the measured shuttle current was interpolated for three voltage levels $2.42,2.4$ and $2.35 \mathrm{~V}$ and it is presented in Fig. 10, from where the trend is clearly visible. The period of the shuttle current growth between 0 and 120 cycles can be linked to the fast capacity fade processes. Afterward, the shuttle current reaches its maximum and starts to decline, having a shape similar to that of the degrading discharging capacity. It is worth to notice that the shuttle current trend is similar to various voltage levels. Therefore, only one voltage value could be considered sufficient for the shuttle current measurements in terms of tracking the degradation.

Looking at the cell's resistance, shown in Fig. 9c), and power capability, shown in Fig. 9d), they remained relatively constant over the high and the medium SOC region (except the last point at 400 cycles). Only at low SOC, the resistance exhibits a higher increase, while a near proportional decrease is obtained for the power capability. Over the aging period, one can see that the resistance and power curves are moving at first to the left (120-240 cycles), where the inflection point between the high and low voltage plateaus is moving toward a higher SOC. Afterward (280-400 cycles), the curves (perceived for example again by the inflection point) are moving back to the right. Because the SOC is a relative quantity related to the measured capacity during the capacity measurement, this "curves movement" is rather a consequence of a changing ratio between the high and the low voltage plateaus, which is a result of degradation mechanisms. It is also worth noticing that the particular shape of the resistance profile is strongly correlated with the resistance of the electrolyte. In brief, the electrolyte resistance is dependent on the viscosity of the electrolyte, which is governed by the nature and concentration of different soluble polysulfide species being present in the electrolyte at different states of discharge. ${ }^{2}$

For TC2, shown in Fig. 11a), the capacity fade has a similar evolution as in TC1 until 120 cycles and then the degradation is accelerated. In Fig. 11b) is shown that the shuttle current increased during aging approximately from $0.0559 \mathrm{~A}$ to $0.0881 \mathrm{~A}$ at $2.42 \mathrm{~V}$, which is significantly less than in the case of TC1. Especially in Fig. 11c), it is illustrated that the resistance after an initial drop, has increased during the aging mainly over the medium SOC values (i.e., 20-70\% SOC).

The thickness of the cells was measured at the discharged state after the last RPT. The photos of the cells are shown in Fig. 12. As expected, the thickness of the cells expanded and is larger than the one of the not used cell. However, there is a difference in a shape of the aged cells. The cell tested under TC1 conditions resulted into concavelike 'solid' swelling, which is characterized by a thinner central area of the cell and a thicker outer (side) area. The cell aged under TC2 conditions resulted into a shape described as 'gaseous' swelling, but because the cell felt hard by touch, it is assumed that the shape was determined by changes in the solid structure and not by gassing, which matches with results seen in Ref. 22. From these results is apparent that the mechanical structure of the aged cell is influenced by the discharging current amplitude.
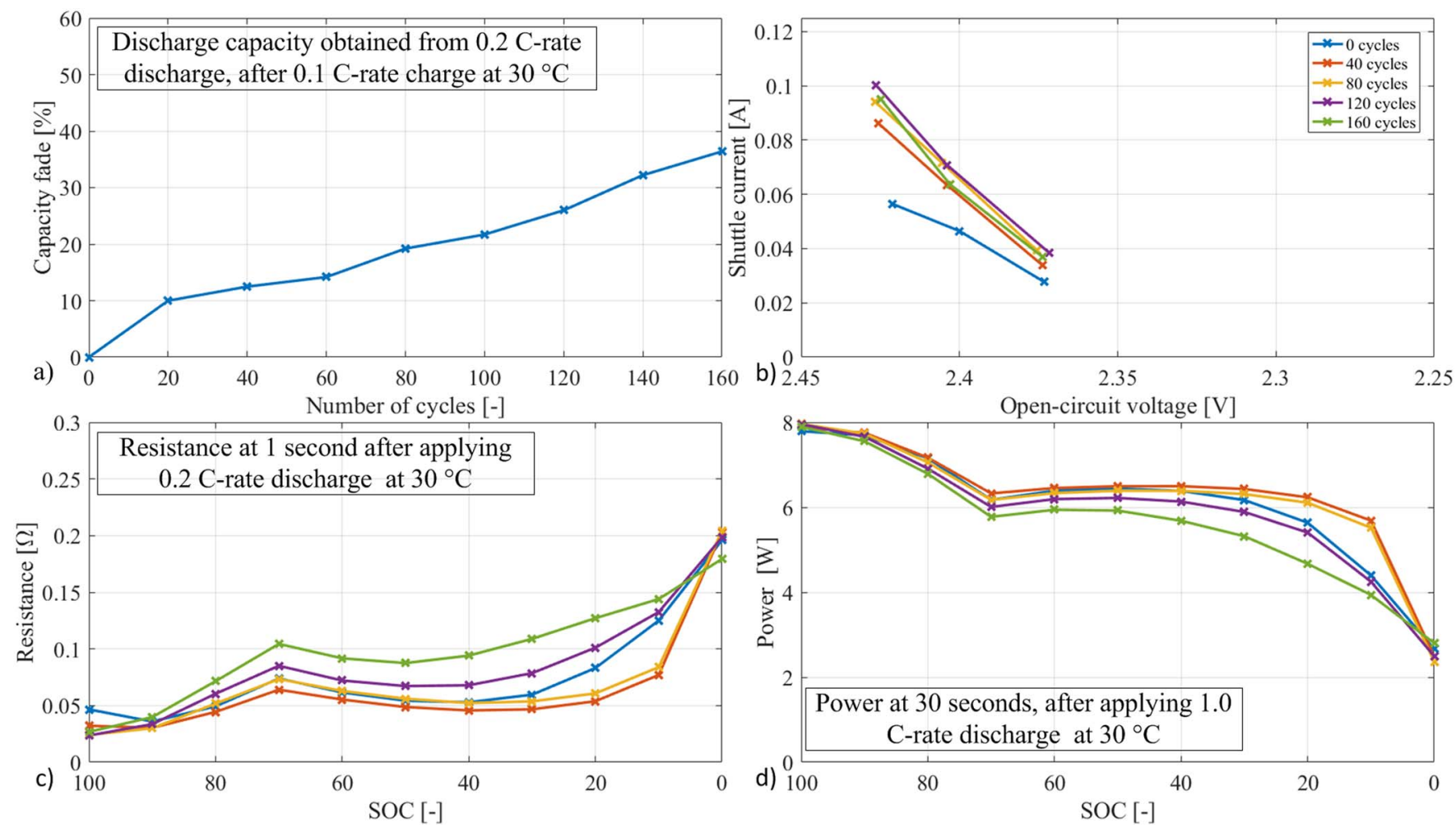

Figure 11. Evolution of the performance parameters obtained during TC2 aging ( $0.1 \mathrm{C}$-rate charging to $2.45 \mathrm{~V}$ or 11 hours, $0.5 \mathrm{C}$-rate discharging to $1.5 \mathrm{~V}$ at $\left.30^{\circ} \mathrm{C}\right)$. 


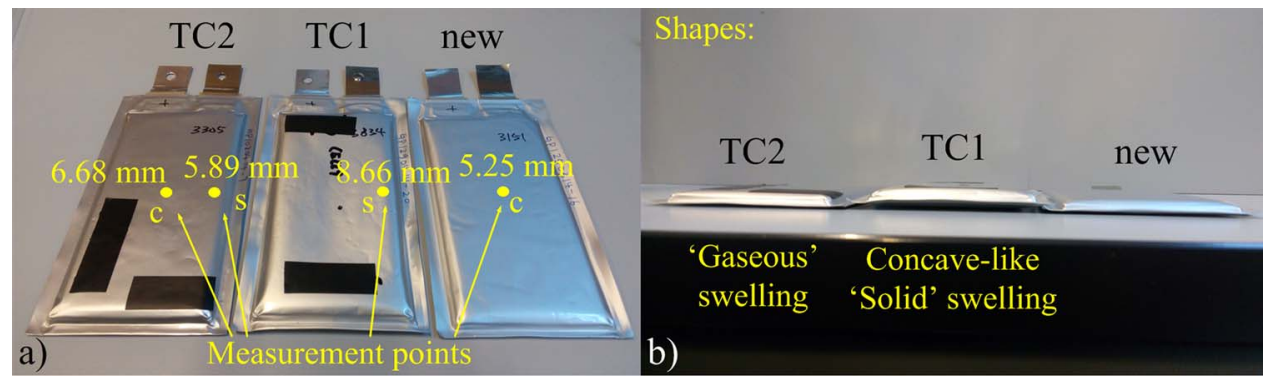

Figure 12. Photos of the used 3.4 Ah OXIS Energy Li-S cells. a) Front view with illustrated measurement points, where $c$ is for center and $s$ is for side, and the value of the cell thickness at that point. b) Side view of the cells and their identified shape as defined in Supplementary data of Ref. 22.

\section{Conclusions}

Li-S batteries with their unique behavior require an improved testing approach, where the suitability of methods applied for Li-ion batteries should be carefully assessed and tailored. Often, it is not possible to directly apply proven approaches from the world of $\mathrm{Li}$-ion batteries, such as modeling and testing, and apply them to the Li-S batteries since this would cause biased results and significant loss of accuracy. Therefore, an RPT procedure for the Li-S batteries is proposed in this manuscript, to bring attention to the specific issues and differences of this type of batteries and to provide guidance to other users. The RPT is typically used to evaluate the performance of the batteries related to the practical applications and often it is applied to identify the influence of aging at different conditions.

The use of the proposed RPT procedure on the aging of Li-S cells shown that the cells cycled at different conditions age in a different way, which has also a different effect on their performance parameters such as capacity, shuttle current, resistance and power capability. Therefore, it is important to apply for lifetime tests a test methodology, which is able to capture the evolution of all these parameters. Moreover, the obtained results can be used to study degradation mechanisms of Li-S cells. It is expected that for future $\mathrm{Li}-\mathrm{S}$ cells or $\mathrm{Li}-\mathrm{S}$ cells oriented to power performance, the C-rates can be adjusted and increased, especially for the nominal cycle, which will reduce the necessary testing time demand.

\section{Acknowledgments}

This work has been part of the ACEMU-project. The authors gratefully acknowledge the Danish Council for Strategic Research (1313-00004B) and EUDP (1440-0007) for providing financial support and thank OXIS Energy for supplying the Lithium-Sulfur battery cells.

\section{ORCID}

V. Knap (D) https://orcid.org/0000-0003-0108-1714

D-I. Stroe (1D) https://orcid.org/0000-0002-2938-8921

\section{References}

1. D. Bresser, S. Passerini, and B. Scrosati, Chem. Commun., 49, 10545 (2013).

2. M. Wild, L. O'Neill, T. Zhang, R. Purkayastha, G. Minton, M. Marinescu, and G. J. Offer, Energy Environ. Sci, 8, 3477 (2015).

3. I. a. Hunt, Y. Patel, M. Szczygielski, L. Kabacik, and G. J. Offer, J. Energy Storage, 2, 25 (2015).

4. G. Xian-Zhong, H. Zhong-Xi, G. Zheng, Z. Xiong-Feng, L. Jian-Xia, and C. Xiao-Qian, Aircr. Eng. Aerosp. Technol., 85, 293 (2013).

5. ISO 12405-1:2011 Electrically propelled road vehicles-Test specification for lithium ion traction battery packs and systems-Part 1: High-power applications, (2011).

6. ISO 12405-2:2012 Electrically propelled road vehicles-Test specification for lithiumion traction battery packs and systems-Part 2: High-energy applications, (2012).

7. IEC 62660-1: Electrically propelled road vehicles - Test specification for lithium-ion traction battery packs and systems - Part 1: High power applications, (2011).
8. IEC 62660-2: Secondary batteries for the propulsion of electric road vehicles - Part 2: Reliability and abuse testing for lithium-ion cells, (2011).

9. G. Mulder, N. Omar, S. Pauwels, F. Leemans, B. Verbrugge, W. De Nijs, P. Van Den Bossche, D. Six, and J. Van Mierlo, J. Power Sources, 196, 10079 (2011).

10. The Idaho National Laboratory, Battery Test Manual For Plug-In Hybrid Electric Vehicles, (2012)

11. D.-I. Stroe, M. Swierczynski, A.-I. Stan, R. Teodorescu, and S. J. Andreasen, IEEE Trans. Ind. Appl., 50, 4006 (2014).

12. H. He, B. Liu, A. Abouimrane, Y. Ren, Y. Liu, Q. Liu, and Z.-S. Chao, J. Electrochem. Soc., 162, A2195 (2015).

13. M. Marinescu, T. Zhang, and G. J. Offer, Phys. Chem. Chem. Phys., 18, 584 (2016).

14. Q. Wang, J. Zheng, E. Walter, H. Pan, D. Lv, P. Zuo, H. Chen, Z. D. Deng, B. Y. Liaw, X. Yu, X. Yang, J.-G. Zhang, J. Liu, and J. Xiao, J. Electrochem. Soc., 162, A474 (2015).

15. D. Moy, A. Manivannan, and S. R. Narayanan, J. Electrochem. Soc., 162, A1 (2014).

16. M. R. Kaiser, J. Wang, X. Liang, H.-K. Liu, and S.-X. Dou, J. Power Sources, 279, 231 (2015).

17. H. Yao, G. Zheng, P.-C. Hsu, D. Kong, J. J. Cha, W. Li, Z. W. Seh, M. T. McDowell, K. Yan, Z. Liang, V. K. Narasimhan, and Y. Cui, Nat. Commun., 5, 3943 (2014).

18. X. Li, M. Rao, D. Chen, H. Lin, Y. Liu, Y. Liao, L. Xing, and W. Li, Electrochim. Acta, 166, 93 (2015)

19. E. Peled, M. Goor, I. Schektman, T. Mukra, Y. Shoval, and D. Golodnitsky, J. Electrochem. Soc., 164, A5001 (2017).

20. J. Brückner, S. Thieme, H. T. Grossmann, S. Dörfler, H. Althues, and S. Kaskel, J. Power Sources, 268, 82 (2014)

21. Y. Diao, K. Xie, S. Xiong, and X. Hong, J. Electrochem. Soc., 159, A1816 (2012).

22. S. Waluś, G. Offer, I. Hunt, Y. Patel, T. Stockley, J. Williams, and R. Purkayastha, Energy Storage Mater. (2017).

23. Z. Deng, Z. Zhang, Y. Lai, J. Liu, J. Li, and Y. Liu, J. Electrochem. Soc., 160, A553 (2013).

24. A. F. Hofmann, D. N. Fronczek, and W. G. Bessler, J. Power Sources, 259, 300 (2014).

25. S. Risse, S. Angioletti-Uberti, J. Dzubiella, and M. Ballauff, J. Power Sources, 267, 648 (2014).

26. M. Dubarry, V. Svoboda, R. Hwu, and B. Yann Liaw, Electrochem. Solid-State Lett., 9, A454 (2006).

27. V. Knap, T. Kalogiannis, R. Purkayastha, S. Beczkowski, D.-I. I. Stroe, E. Schaltz, and R. Teodorescu, ECS Trans., 77, 1919 (2017).

28. B. Wu, V. Yufit, Y. Merla, R. F. Martinez-Botas, N. P. Brandon, and G. J. Offer, J. Power Sources, 273, 495 (2015).

29. Y.-X. Yin, S. Xin, Y.-G. Guo, and L.-J. Wan, Angew. Chemie Int. Ed., 52, 13186 (2013).

30. V. Knap, D. I. Stroe, M. Swierczynski, R. Purkayastha, K. Propp, R. Teodorescu, and E. Schaltz, J. Power Sources, 336, 325 (2016).

31. https://oxisenergy.com, accessed on 30.4.2018.

32. F. Y. Fan, W. C. Carter, and Y.-M. Chiang, Adv. Mater, 27, 5203 (2015).

33. V. Knap, D.-I. Stroe, M. Swierczynski, R. Teodorescu, and E. Schaltz, J. Electrochem. Soc., 163, A911 (2016).

34. V. Knap, T. Zhang, D. I. Stroe, E. Schaltz, R. Teodorescu, and K. Propp, ECS Trans., 74, 95 (2016)

35. T. Zhang, M. Marinescu, S. Walus, P. Kovacik, and G. J. Offer, J. Electrochem. Soc., 165, A6001 (2018).

36. T. Zhang, M. Marinescu, S. Walus, and G. J. Offer, Electrochim. Acta, 219, 502 (2016).

37. P. G. Bruce, S. A. Freunberger, L. J. Hardwick, and J.-M. Tarascon, Nat. Mater., 11, 19 (2012).

38. H. Noh, J. Song, J.-K. Park, and H.-T. Kim, J. Power Sources, 293, 329 (2015).

39. S. Thieme, J. Brückner, A. Meier, I. Bauer, K. Gruber, J. Kaspar, A. Helmer, H. Althues, M. Schmuck, and S. Kaskel, J. Mater. Chem. A, 3, 3808 (2015).

40. S. Risse, N. A. Cañas, N. Wagner, E. Härk, M. Ballauff, and K. A. Friedrich, J. Power Sources, 323, 107 (2016) 
2018-08-25

Reference performance test

Methodology for degradation assessment of lithium-sulfur batteries

Knap, Vaclav

Electrochemical Society

Knap V, Stroe D-I, Purkayastha R, et al., (2018) Reference performance test Methodology for degradation assessment of lithium-sulfur batteries. Journal of The Electrochemical Society, Volume 165, Issue 9, 2018, pp.1601-1609

https://doi.org/10.1149/2.0121809jes

Downloaded from Cranfield Library Services E-Repository 